\title{
Colour Obsessions and Phobias in Autism Spectrum Disorders: the Case of J.G
}

\author{
Amanda K. Ludlow ${ }^{\mathrm{a}^{*}}$, Pamela Heaton ${ }^{\mathrm{b}}$, Elisabeth Hill ${ }^{\mathrm{b}}$ \& Anna Franklin ${ }^{\mathrm{c}}$ \\ ${ }^{a}$ School of Psychology, University of Birmingham, Birmingham, UK, ${ }^{b}$ Department of Psychology, Goldsmiths College, \\ London, UK, ${ }^{\mathrm{c}}$ School of Psychology, University of Sussex, Brighton, UK
}

\begin{abstract}
The current study is the first investigation of colour 'obsessions' and 'phobias' in ASD. We investigate the colour perception and cognition of J.G., a boy with ASD who has a strong obsession with blue, and a strong phobia of other colours. J.G.'s performance on a series of colour tasks (colour-entity association; chromatic discrimination; colour classification) is compared to 13 children with and without autism who do not have colour obsessions or phobias. The findings lead to the formalisation of two hypotheses: (i) colour obsessions and phobias in individuals with ASD are related to an unusually strong ability to associate colours with entities; (ii) colour obsessions are related to hyposensitivity, and colour phobias to hypersensitivity, in the affected regions of colour space.
\end{abstract}

Key words: Autism Spectrum Disorders, Colour, Obsessions, Phobias

\section{Introduction}

In order for a diagnosis of Autism Spectrum Disorder (ASD) to be made, individuals must have deficits in socialisation, communication and imagination before they are three (DSM-IV, 1994; DSM-1V, TR, 2000). Abnormal responses to sensory stimuli were omitted from DSM-III (1980) because of confusion over the interpretation of such symptoms (Ornitz, 1989), and also because of the poverty of systematic empirical research in this domain (Filipek et al., 1999). Yet since DSM-IV was published in 1994, there has been increasing awareness that sensory processing difficulties may be an important symptom in the clinical picture, for many, if not most individuals with autism (e.g., Dawson \& Watling, 2000; Leekam, Libby, Wing, \& Gould, 2007; Liss, Saulnier, Fein, \& Kinsbourne, 2006; Minshew \& Hobson, 2008).

Case studies are important in enabling the exploration of idiosyncratic sensory behaviours, frequently reported in both anecdotal accounts (e.g., Grandin, 1995; Jones, Quigney, \& Huws, 2003) and behavioural studies of individuals with autism (Baranek, Boyd, Poe, David \& Watson, 2007; Tomchek \& Dunn, 2007). In the current study we investigate the case of a male child with autism (J.G.) who has a longstanding obsession for the colour blue and limited tolerance for most other colours. There have been previous anecdotal reports of children with autism having 'colour obsessions' and 'colour phobia'. For example, in one case, a mother discusses her child's obsession with red: "By and large he would eat only red food, and to this day he uses ketchup to mask unwelcome colours" (Moore, 2004, p.153). These anecdotal reports appear to be common, although the prevalence of colour obsessions and phobias in ASD is currently unknown, and to the best of our knowledge, colour obsessions and phobias in ASD have yet to be systematically investigated. Given the behavioural consequences of such a phenomenon, it is surprising that very little is known about the causes and correlates of colour obsessions and phobias, or the individuals who display them (White \& White, 1987; Williams, 1999). The current investigation makes a first step in understanding the phenomena, and paves the way for large scale investigations in the future.

\subsection{Case Report on J.G.}

J.G. was first diagnosed with "High Functioning Autism" (HFA) in 1999 when he was 4 years 6 months. The diagnosis was carried out by a consultant and Senior Lecturer in Child and Adolescent Psychiatry at an Autism Spectrum Disorders Clinic that was part of a UK Child and Family Mental Health Service. In a subsequent assessment carried out by a paediatrician and an educational psychologist, "four out of nine features of inattention, five of five features of hyperactivity, and four of four features of impulsivity on a diagnostic checklist" were noted as atypical. Consistent with his diagnosis of autism, J.G.'s language onset was significantly delayed, with his first attempt to speak occurring around four years. When J.G.'s cognitive ability was assessed at 10.5 years, the Raven's Progressive Matrices (Raven, Court \& Raven, 1992), a test of fluid, non-verbal intelligence, yielded a scaled score of 103. Further assessment using the Weschler Intelligence Scales for Children Revised (WISC-R) ( $3^{\text {rd }}$ edition) (Wechsler, Golombok \& Rust, 1992) showed that Full Scale IQ was 104, Performance IQ was 105 and Verbal IQ was 102. Whilst these assessments do not indicate global intellectual impairment, the pattern of performance across the subtests of the WISC revealed an extremely uneven ability profile. He performed well on the tasks assessing general knowledge and understanding of information (information scaled score $=14$ and similarity scaled score $=14$ ), but poor performance on tasks requiring a higher level of verbal explanation (vocabulary scaled score $=7$ and comprehension scaled score $=1)$.

J.G. has very strong reactions to many different types of sensory input, including colour obsessions and phobias. Since early childhood, J.G. has had an aversion to bright colours, 
and a very marked preference for blue and purple. His family have reported early incidents during which he attempted to "dye" clothes and other objects blue. Indeed, he even attempted to paint the family dog this colour. Within the family home, J.G.'s environment has been tailored to reduce his stress. To this end, his bedroom is painted purple and black, all of his clothes are dark blue, and the family car is blue with a purple interior. Similar to cases previously reported in the literature (Moore, 2004), J.G's obsession with colour impacts upon his diet. He opts for foods that are either white or have little colour and will not tolerate more than two colours on a plate at any one time. J.G. reports pain in his eyes ("hot eyes") in responses to bright colours, and exposure to these results in hyper-excitability and nausea (Ludlow \& Wilkins, 2009). He dislikes all types of light, and his bedroom remains unlit. Although his tolerance for many bright colours has improved with age, he continues to carry his sunglasses around with him, even indoors. J.G. is also unable to tolerate strong smells, for example the smell of washing powder and glue. His auditory difficulties include a marked intolerance to the sound of vacuum cleaners. When assessed on the Sensory Profile Questionnaire (Dunn, 1999), a questionnaire which provides a standard method to measure a child's sensory processing in daily life, J.G is atypical on 7 of the 9 factors (see table 1). The comparison of J.G's profile with the ASD norms (Dunn, 1999) suggest that J.G's sensory difficulties are more extreme than is typical in ASD.

Table 1. Mean scores across the nine factors of the Sensory Profile for J.G., ASD and TD groups.

\begin{tabular}{cccc}
\hline Factors & J.G. & ASD & TD \\
\hline Sensory Seeking $(\max =85)$ & $38^{*}$ & 55 & 72 \\
Emotionally Reactive $(\max =80)$ & $44^{*}$ & $44^{*}$ & 65 \\
Low Endurance/Tone $(\max =45)$ & $32^{*}$ & $34^{*}$ & 42 \\
Oral Sensory Sensitivity & $21^{*}$ & 30 & 38 \\
$\quad(\max =45)$ & & & \\
Inattention/Distractibility & $10^{*}$ & $20^{*}$ & 28 \\
$\quad(\max =35)$ & & & \\
Poor Registration $(\max =40)$ & $26^{*}$ & $26^{*}$ & 36 \\
Sensory Sensitivity $(\max =20)$ & $9 *$ & 15 & 15 \\
$\quad$ Sedentary $(\max =20)$ & 11 & 13 & 13 \\
Fine Motor/Perceptual $(\max =15)$ & 9 & $7^{*}$ & 12 \\
\hline
\end{tabular}

* Atypical Performance - based on means taken from the Sensory Profile (Dunn, 1999). Lower scores represent atypical sensory processing.

\subsection{Colour Perception and Cognition in a Child with Colour Obsessions and Phobias}

The current investigation aims to establish whether J.G.'s colour obsessions and phobias are accompanied by atypical characteristics for other aspects of colour perception and cognition. In a series of three experiments we compare J.G.'s performance to ASD and TD groups of children who do not have colour obsessions or phobias, on tasks that measure the ability to associate colour with entities (Experiment 1), the accuracy of chromatic discrimination (Experiment 2), and the pattern of colour classification (Experiment 3). The theoretical motivation for the investigation of these aspects of colour perception and cognition in J.G. is outlined in the introduction to each experiment. The study represents the first step in understanding the underlying mechanisms, causes and consequences of colour obsessions and phobias in ASD. It is intended that this case study provides a springboard for future large scale investigations of colour obsessions and phobias in those with ASD. Full ethical approval was granted to complete these studies and all children and their parents' gave their informed consent prior to their/their child's inclusion in the study.

\section{Experiment 1. Colour Associations}

Experiment 1 aims to establish whether J.G. is atypical in his ability to associate entities with colour. One recent theory of colour preference argues that colour preferences arise from colours being associated with valenced objects (Palmer \& Schloss, 2010). For example, it is argued that we like colours that are associated with liked objects, and dislike colours that are associated with disliked objects. If this is the case, then it is possible that the strong colour preferences and aversions to colours seen in J.G. and other individuals with ASD, could be related to a superior ability to associate entities, such as objects or even experiences, with colours. For example, a favourite toy may be more readily associated with its colour, leading to a stronger colour preference than is typically the case. To investigate whether J.G has a superior ability to associate colours and entities, we assess his ability to learn and retrieve arbitrary colour-animal associations relative to groups of both typically developing children (TD) and children with ASD. The task we use is taken from the first phase of an experiment in Heaton, Ludlow and Roberson (2008), where no differences between groups of children with and without ASD were found when children were matched on non-verbal cognitive ability (when both groups had learning difficulties). The simple task involves children remembering and retrieving arbitrary associations of colours and animals.

\subsection{Participants}

In addition to J.G., groups of typically developing children (TD, $\mathrm{N}=13$ ) and children with autism (ASD, $\mathrm{N}=13$ ) were also tested. Samples were matched to J.G.'s age and non-verbal cognitive ability. The TD group had mean age of 10 years, 8 months ( $\mathrm{SD}=2.4)$ and a mean Ravens Matrices score of 89.1 $(\mathrm{SD}=14.4)$ and were recruited from two mainstream primary and secondary schools. The ASD group had a mean age of 10 years 9 months $(\mathrm{SD}=2.7)$ and a Ravens Matrices score of 90.5 (SD = 10.6). The thirteen children with ASD (5 with a diagnosis of Asperger's Disorder and 8 with a diagnosis of Autism) were recruited from a specialist school, to which entry is dependent upon meeting criteria for autism spectrum disorders outlined in DSM IV (APA, 1994). All participants were screened for colour vision deficiencies using the City University Colour Test ( ${ }^{\text {rd }}$ Edition, Fletcher, 1998) and the Ishihara Test (Ishihara, 1970). No participants, including J.G, showed any colour vision defects. In order to screen out any other potential cases of colour obsession, all children were questioned about colour preferences. Parents also filled in a short colour preference questionnaire on their child's behalf (Appendix) addressing their children's colour likes and dislikes. Children who were shown to have strong colour preferences for a reasonable duration of time $(1$ year +$)$ and, more importantly, also changed their behaviour accordingly (e.g, only wears a certain colour), were not included in the experimental trials. Two children with autism were excluded due to showing a strong colour preference, in this case one 
liked orange and the other liked red, but no controls were excluded.

\subsection{Stimuli and Set up}

Four hues were taken from the Munsell colour system (red: 5R; blue; 5B; green: 5G; yellow: $5 \mathrm{Y}$ ) at constant value (6) and chroma (6). The CIE (1931) x,y,Y chromaticity coordinates of these Munsell colours were rendered as $3 \mathrm{~cm}^{2}$ patches on a 17 inch CRT computer monitor ${ }^{1}$. Animal pictures were black and white $7 \mathrm{~cm}^{2}$ images of a dog, cat, rabbit and pig which were taken from the Snodgrass and Vanderwaart (1980) picture scale. Participants sat approximately $49 \mathrm{~cm}$ from, and at eye-level to, the computer monitor on which stimuli were presented.

\subsection{Design and Procedure}

Participants were told that they would see four animals paired with four colours. They were advised that they would be asked to remember which animal and colour were paired with each other. During the familiarisation phase, one of four colour-animal pairings (red-dog; blue-cat; yellow-rabbit; green-pig) was shown on each trial, with the coloured stimulus presented in the centre of the monitor, and the animal stimulus presented $2 \mathrm{~cm}$ below the colour. There were 16 randomised familiarisation trials, four for each pairing. Then, during a test phase, one of the four animals appeared on each trial, with the four coloured stimuli presented in a horizontally aligned arrangement below (1-4 position of stimulus randomised). Participants were asked to point to the colour which was paired previously with the animal currently seen. There were 16 randomised test phase trials, four for each animal.

\subsection{Results}

The accuracy of performance on the task is collapsed across colour regions, as the accuracy in each colour region could be influenced by the specific colour-animal association allocated to it. Figure 1 gives the percentage accuracy in identifying the colour-animal association during the test phase for J.G. and TD and ASD groups. There was no significant difference in accuracy for TD and ASD participants, $t(24)=$ $1.40, p=0.18$.

As can be seen in figure 1, J.G. identified all colouranimal associations correctly. Six other participants (2 ASD and 4 TD) were also $100 \%$ accurate on the task, and J.G.'s performance was not significantly different to the other participants (ASD and TD combined: $Z=1.33)$, or ASD $(Z=$ $1.75)$ or TD groups $(Z=1.02)$.

As in Heaton et al., the ASD group had no particular strength or deficit in associating an animal with blue, yellow, green or red, relative to controls matched on non-verbal cognitive ability. J.G. is also not significantly different in his performance on this task relative to ASD and TD groups, although he does score perfectly on the task.

Ceiling effects for J.G on the task could be masking the extent of his ability, and with a more difficult task, his performance may well have been significantly different to the ASD and TD groups.
Figure 1. Mean accuracy (+/-1se) in identifying colouranimal associations for J.G. and TD and ASD groups.

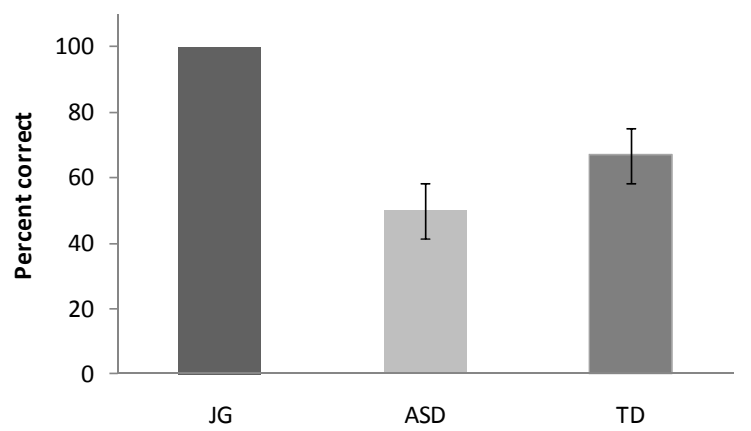

2.5. Discussion

However, although we cannot say with confidence whether J.G.'s performance is significantly different to ASD and TD groups, his perfect performance on the task does clearly suggest that he is excellent at associating colours with entities. The findings therefore do not contradict the hypothesis that strong colour obsessions and aversions in ASD may result, in part, from a particularly strong ability to associate colours with entities. If this hypothesis is correct, it would suggest that colour obsessions and phobias in ASD derive from similar underlying mechanisms to those that underlie colour preferences and aversions in typical development, but that the underlying mechanisms are amplified. Of course, a strong ability to form colour-entity associations could also be a consequence rather than a cause of colour obsessions. For example, strong reactions to colour could draw attention to the colour of objects, thereby strengthening the ability to form or retrieve these colourentity associations. Experiment 1 suggests that it is warranted to further investigate these issues in future larger scale investigations of the underlying mechanisms of colour obsessions in ASD.

\section{Experiment 2. Discrimination: finding the chromatic 'odd- one-out'}

An alternative hypothesis to the colour-association hypothesis is that children with colour obsessions and phobias are atypical in their sensitivity to colour and chromatic differences in colour (Franklin \& Sowden, 2011). For example, a general hypersensitivity to colour could lead to greater recognition of the nuances of different colours, and could potentially elicit stronger reactions as a result. Alternatively, chromatic hypersensitivity could result from the colour obsessions and phobias themselves. For example, the greater visual experience with preferred colour regions, as a result of the structuring of the chromatic environment to alleviate stress (e.g., ensuring bedroom walls and other artefacts are the preferred colour), could lead to hypersensitivity in these chromatic regions as a result of experience induced perceptual learning (e.g., Fahle \& Poggio, 2002).

Previous investigation of colour perception and cognition in children with ASD has provided converging evidence that chromatic discrimination is reduced rather than enhanced in 'high-functioning' adolescents with ASD relative to age and non-verbal cognitive ability matched controls, at least under some circumstances (Franklin, Sowden, Burley \& Notman, 
2008; Franklin et al., 2010; Fujita, Yamaski, Kamio, Hirose \& Tobimatsu, 2011). For example, Franklin et al. (2008, 2010) have found that ASD participants were on average less accurate at memorising and searching for coloured targets, had poorer performance on a clinical test of colour vision (the Farnsworth-Munsell 100 hue test), and had elevated chromatic discrimination thresholds (yet performed at typical levels on these tasks when stimuli varied along dimension other than colour). Although Koh, Milne and Dobkins (2011) find no evidence for atypical contrast sensitivity to moving chromatic gratings in ASD, visual evoked potentials elicited by static chromatic gratings have been found to be reduced in those with ASD relative to controls (Fujita et al., 2011) ${ }^{2}$. Despite such group differences on chromatic tasks, it remains possible that the individuals with colour obsessions and phobias are in fact hypersensitive to chromatic differences throughout colour space, or that this hypersensitivity is selective to regions of colour space that are the focus of the obsession or phobia (see Franklin \& Sowden, 2011 for further discussion).

Experiment 1 assesses this, by comparing J.G.'s performance on a simple chromatic discrimination task for preferred and disliked regions of colour space, to the performance of groups of TD and ASD participants who do not have colour obsessions or phobias. The task is taken from a previous investigation of colour perception in ASD (Heaton et al., 2008), and involves identifying the colour that is different out a set of three coloured stimuli. Heaton et al. found poorer performance on the task for a group of ASD children who had delays in verbal and non-verbal cognitive ability relative to a TD group matched on chronological age, yet equivalent performance to a group of children matched on verbal and non-verbal cognitive ability. Here, we compare J.G.'s performance of the task to TD and ASD groups of participants who are matched on chronological age and nonverbal cognitive ability.

\subsection{Participants}

The participants were the same as in Experiment 1.

\subsection{Stimuli and Set up}

Stimuli were 32 hues taken from the Munsell colour system at constant value (6) and chroma (6). The 32 hues were from four regions of colour space (8 each from red, green, yellow, blue) and had hue codes $(1,9.5,10)$ for training stimuli, and hue codes $(2.5,5,7.5$ and 10) for test phase stimuli. All other aspects of set up were identical to Experiment 1 .

\subsection{Design and Procedure}

On each trial, three coloured $7 \mathrm{~cm}^{2}$ coloured patches were presented on a gray background (Munsell N5), with each stimulus located at the end points of an imagined equilateral triangle, with the allocation of stimuli to each location randomised. Two of the three stimuli were an identical hue, and the third was different in hue, with both hues from the same colour region (red, green, yellow or blue). Participants were required to identify the different stimulus by pointing. During a training phase, stimulus triads with hues that varied in 8.5 or 9 hue units were presented (e.g., target $=1 \mathrm{~B} 6 / 6$, distractors $=9.5 \mathrm{~B} 6 / 6$ or target $=1 \mathrm{~B} 6 / 6$, distractors $=10 \mathrm{~B} 6 / 6)$. There were 6 randomised training trials, with 2 for each colour region. During the test phase, the target and distractors varied either by $2.5,5$ or 7.5 hue units). There were 24 randomised test phase trials, with 6 trials for each colour region.

\subsection{Results}

Figure 2 gives the percentage of trials on which the target stimulus was correctly chosen, for each of the four colour regions, for J.G and ASD and TD groups.

Figure 2. Mean accuracy (+/-1se) in identifying the chromatically different stimulus for blue, red, yellow and green regions of colour space, for J.G. and TD and ASD groups.

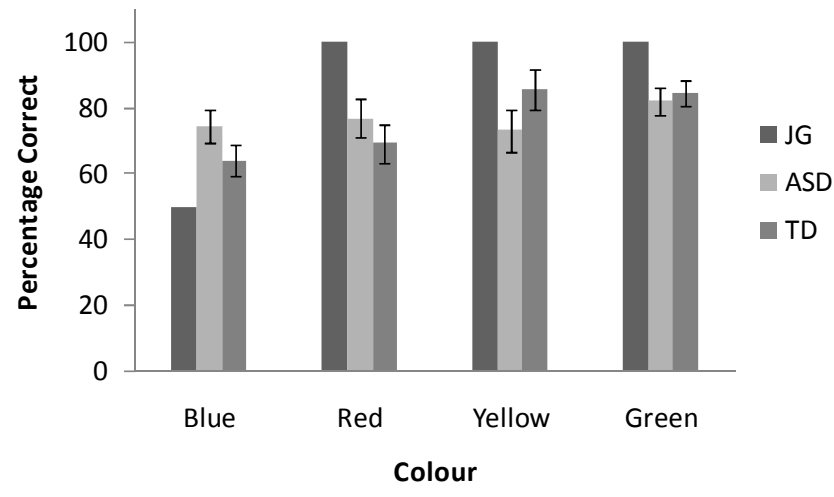

Two way repeated measures ANOVA with Colour Region (Blue/Red/Yellow/Green) and Group (ASD/TD) identified that performance varied across colour regions for TD and ASD groups, $F(3,72)=3.78, p<.05$, but in different ways for each group, $F(3,72)=6.29, p<.005$. There were no significant differences in accuracy of target identification for ASD and TD groups, $(F<1)$. J.G.'s overall accuracy on the task (mean $=87.5 \%)$ was not significantly different to that of ASD and TD groups (overall mean $=71.26 \%, \mathrm{SD}=30.1$ ), $\mathrm{Z}=$ 0.88 . As can be seen in the figure 2, J.G. made no mistakes for three of the colour regions, but was only correct half of the time at identifying the different hue when hues are taken from his preferred colour region. Other participants also made no errors in identifying blue $(\mathrm{N}=2)$, red $(\mathrm{N}=2)$, yellow $(\mathrm{N}=2)$ and green $(\mathrm{N}=8)$, and J.G.'s performance is not significantly different to ASD and TDs in any of the colour regions: blue, $\mathrm{Z}$ $=-1.06$; red, $Z=1.71$; yellow, $Z=-0.56$; green, $Z=1.18$ ). However, J.G.'s distribution of errors across the four colour regions does appear to be atypical: out of 26 participants, only one other participant has errors restricted to one colour region (and that participant only makes one error). Therefore, J.G.'s pattern of errors does suggest that he has a selective deficit in discrimination in the region of colour space that he prefers ${ }^{3}$. This argument is also strengthened by the evidence that J.G.'s performance is greater than the ASD and TD mean accuracy for red, yellow and green, yet below the ASD and TD mean accuracy for blue.

\subsection{Discussion}

The finding of equivalent levels of performance for those with ASD to groups of children matched on non-verbal cognitive ability aligns with the previous finding of Heaton et al. who found that children with learning difficulties did not differ on the task depending on whether or not they had ASD. The current study indicates that children with ASD perform at typical levels when identifying the stimulus that is 
chromatically different. However, on the basis of converging evidence from previous research that chromatic discrimination in ASD is reduced (e.g., Franklin et al., 2008; 2010; Fujita, Yamasaki, Kamio, Hirose \& Tobimatsu, 2011), one may expect those with ASD to perform less well than TD participants on this task. One possibility is that the current task is not sensitive enough to reveal the differences in chromatic discrimination between the groups. For example, previous research has established that although the deficit in chromatic discrimination in ASD is reliable, it is indeed small (e.g., the difference in chromatic just-noticeable-difference between ASD and TD groups is small but significant: Franklin et al., 2010). The chromatic differences on the current task may well have been too large to reveal differences between the groups - indeed several of the participants had perfect performance in one or more colour regions on the task.

Although J.G's general ability to identify the stimulus that is chromatically different is not statistically different to TD or ASD groups, he does have perfect performance in three colour regions. The task therefore does reveal the interesting atypical distribution of errors across colour regions for J.G. relative to ASD and TD children. Whilst J.G. is performing at the highest possible level for red, green and yellow regions, his performance is below typical levels (as indicated by ASD and TD means) for the blue region of colour space. As outlined in the introduction, J.G. has a strong obsession for blues and purples, and a strong phobia of other regions of colour space. This suggests that J.G. has a selective deficit in chromatic discrimination in his preferred region of colour space, whilst also being excellent at chromatic discrimination in the regions of colour space that he is averse to.

These findings from J.G. lead to our tentative suggestion that chromatic hyposensitivity underlies colour obsessions in ASD, whereas chromatic hypersensitivity underlies colour phobias. It may seem odd to propose that children with ASD like stimuli that they are poor at discriminating, and dislike stimuli that they are excellent at discriminating. However, it is theoretically possible that if an individual is hypersensitive to certain colour regions that this causes an unpleasant 'sensory overload', and that colours from regions of hyposensitivity are sought out to avoid this. For example, J.G.'s experience of 'hot eyes' and excitability in response to bright colours, and his seeking out of less stimulating blue colours could be a real world example of sensory overload impacting on colour preference. Of course, this suggestion is based on data and observations from only one individual with colour obsessions, yet it does provide a clear and testable hypothesis for further research.

\section{Experiment 3. Classification of Colours: Naming and Grouping}

J.G.'s poor performance on the discrimination task when stimuli were in his preferred region of colour space could mean that J.G. has difficulty in processing preferred colours in other ways as well. For example, J.G.'s difficulties in discrimination in the blue region of colour space may impact on his ability to classify colours in and around that colour region. Experiment 3 aims to assess this, and compares J.G.'s naming and grouping of a set of colours ranging from green to purple, to TD and ASD groups.Previous research has established that the naming and categorising of colours is typical in ASD, at least for the blue-green region that was tested (Franklin et al., 2008). Here we assess whether J.G.'s obsessions with blue and purple colours is associated with atypical classification of colours within and around these regions of colour space.

\subsection{Participants}

Experiment 3 compared J.G.'s performance to new groups of TD $(\mathrm{N}=13)$ and ASD children $(\mathrm{N}=13)$. Again, samples were matched to J.G.'s age and non-verbal cognitive ability. The TD group had mean age of 10 years, 5 months $(\mathrm{SD}=1.9)$ and a mean Ravens Matrices score of 87.2 (9.7). The ASD group had a mean age of 11 years 1 month (SD 2.7) and a Ravens Matrices score of 82.7 ( $\mathrm{SD}=9.8)$. As for Experiment 1 and 2, all children were screened for colour vision deficiencies, and questioned about colour preferences and none reported colour obsessions or phobias.

\subsection{Stimuli and Set up}

Stimuli used were taken from the Munsell colour system which has three dimensions: hue, value (lightness), and chroma (roughly equivalent to saturation or colourfulness). There were 18 hues spanning the green-purple region of colour space (5G-7.5P) in steps of 2.5 Munsell hue units, at constant value (5) and chroma (10). Stimuli were cut from reflective Munsell sheets of card in the shape of a boy $(4 \mathrm{~cm}$ long and $2 \mathrm{~cm}$ wide), and were presented on a gray background (Munsell N5; 13×10 cm). The shape was used to make the children focus on the colour. Only boy shapes were used to reflect the all male sample. The procedure was conducted under standard lighting conditions that simulate natural daylight (D65, 6500K, at 810-1880 lux). These lighting conditions are necessary for the uniformity of the Munsell system to be maintained (Davies \& Franklin, 2002).

\subsection{Design and Procedure.}

For the naming task, each of the stimuli were presented individually and participants were asked to name the stimulus as either green, blue or purple. Each stimulus was presented 3 times each, giving 54 trials in total which were presented in a random order. For the grouping task, all stimuli were arranged in a random arrangement and participants were instructed to group the stimuli into green, blue and purple groups. They repeated this task three times.

\subsection{Results and Discussion}

Figure 3 gives the naming and sorting data for J.G, the ASD and TD groups. The graphs in the naming column give the percentage of trials on which a green, blue or purple response was given for each stimulus. The graphs in the sorting column give the percentage of trials on which each stimulus was allocated to the green, blue or purple groups. 
Figure 3 Naming and grouping classification curves for the green-purple region of Munsell hue space, indicating the frequency with which stimuli are named or grouped using green, blue or purple terms, for J.G. and TD and ASD groups. The $\mathrm{x}$ axis gives the frequency of name or group, and the y axis gives the Munsell hue codes for the stimuli.
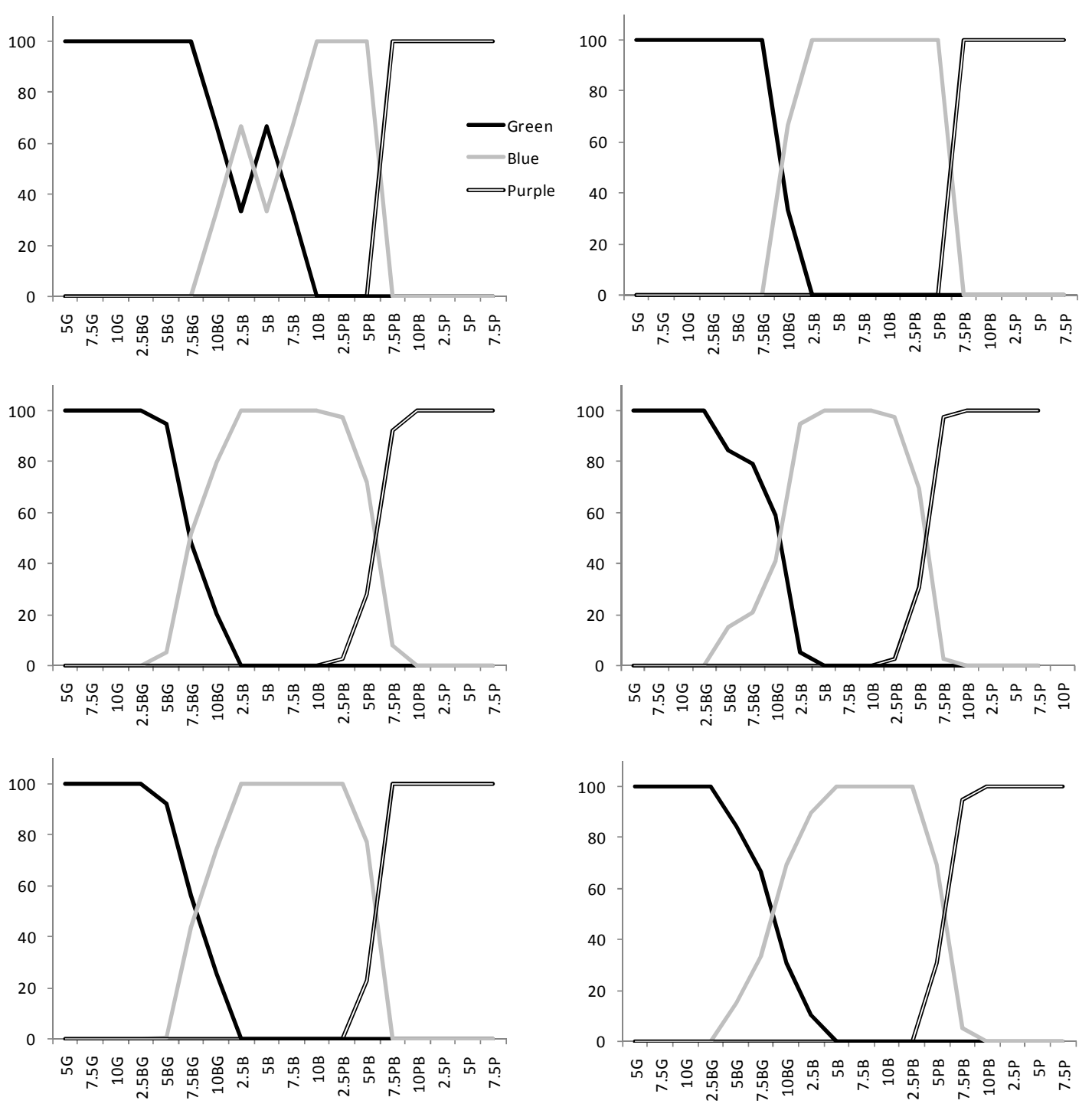

As can be seen in the figure, for ASD and TD groups, the location of the blue-green boundary appears to be between 7.5 and $10 \mathrm{BG}$, and the location of the purple boundary between 5PB and 7.5PB on both tasks. J.G.'s performance aligns with the TD and ASD groups for the blue-purple boundary on both tasks, and the blue-green boundary on the sorting task. However, for the naming task, the blue-green boundary for J.G. shows a different pattern to the TD and ASD groups. For $\mathrm{J} . \mathrm{G}$, there is a region of confusion in naming that spans 7.5 hue units between $10 \mathrm{BG}$ and $7.5 \mathrm{~B}$, where the classification curves do not form a smooth continuum. Although the naming figures for ASD and TD groups below are averaged across participants, no individual in either group displays confusion in naming in this way.

\subsection{Discussion}

Experiment 3 does not provide strong evidence to support the hypothesis that J.G.'s classification of colours in and around his preferred region of colour space is strikingly atypical. J.G.'s classification of colours as either blue or purple is clearly typical relative to ASD and TD groups.

There is some suggestion from the naming task that J.G. may have greater difficulty than ASD and TD groups at classifying colours as either blue or green. This confusion is perhaps surprising as the boundary demarcates colours that he likes (blues) from colours that he dislikes (greens). However, J.G.'s classification of colours in this region would actually have been typical if one of the stimuli $(5 \mathrm{~B})$ was named blue on all three trials rather than just the one. Therefore, as the atypical pattern relies on the naming of just one stimulus, J.G.'s naming in the blue-green region may be less atypical than initially appears. In addition, J.G. is typical at sorting blue and green stimuli, and his blue-green boundary, as indicated by sorting, aligns with ASD and TD groups, suggesting that J.G does indeed have access to the typically elicited terms. Overall then, there is no striking evidence that J.G.'s difficulty at discriminating colours in the blue region as revealed in Experiment 2, is associated with greater confusion when classifying colours within and around this region of colour space. 


\section{General Discussion}

Whilst colour obsessions and phobias in individuals with ASD have been reported anecdotally, this case study provides the first systematic investigation of the phenomenon and of the potential causes and consequences. We intend for the case of J.G. to provide a starting point for understanding this often reported, but under investigated phenomenon. Although conclusions from across the three experiments must be tentative given that they are based on one case study, the current investigation does allow us to formalise two testable hypotheses on colour obsessions and phobias in ASD that can subsequently be tested in large scale investigations. The first hypothesis is that colour obsessions and phobias in ASD result from an amplified ability to associate colours with entities. Convincing evidence has been provided that colourobject associations are related to colour preferences in typical adults (Palmer and Schloss, 2010). The first hypothesis merely suggests that this mechanism is amplified in some individual's with ASD. The case of J.G. potentially provides evidence to support this hypothesis, as unlike the majority of other participants, J.G. never made a mistake when reproducing arbitrary colour-animal associations. An alternative interpretation of J.G.'s performance is that a strong ability to form colour-entity associations is a consequence, not a cause, of colour obsessions and phobias. For example, the strong reactions to colour seen in individuals with colour obsessions and phobias, may lead to them placing more importance on the colours of objects in their environment (e.g., only eating red food, painting the dog blue), thereby strengthening the ability to form colour-entity associations. The current study suggests that further investigation of these issues is warranted, with converging measures of colourentity association, and with a large sample of those with ASD who have colour obsessions and phobias.

The second, testable hypothesis that is formalised from our case study of J.G., is that those with ASD are hyposensitive in colour regions which form the focus of obsessions, yet are hypersensitive in colour regions that are associated with phobic reactions. The argument here is that hypersensitivity and 'sensory overload' in particular regions of the colour space leads to colour phobia, and as a result other less stimulating colour regions are sought out. J.G.'s performance is congruent with this hypothesis, as he only ever makes errors on the chromatic discrimination task when the colours come from his preferred colour region. Again, this evidence at least suggests that further investigation of the hypothesis is warranted. Further research should use more sensitive measures of chromatic discrimination, such as the psychophysical chromatic discrimination threshold task used by Franklin et al. (2010), and test for hyper- and hyposensitivity in regions of colour space associated with obsessions and phobias in a range of individuals.

Further investigation of colour obsessions and phobias in ASD is essential, as it appears that these are common and often have a pervasive impact on behaviour and daily life for those with ASD (Franklin \& Sowden, 2011). In the case of J.G., the colours that he is 'phobic' of elicit a strong physical reaction of nausea. Another case (M.C., a 12 year old boy) has recently been presented to us where an extreme colour obsession with red and phobia to blue also has a pervasive impact on the individual's life (Ludlow \& Wilkins, clinical observation). All of M.C.'s clothes are red, as are his items in his bedroom at home. He shows a physical aversion to touching anything that is blue and he will begin a ritual of obsessively wiping his hands when this happens. These cases, along with other anecdotal reports from the literature (e.g.,Williams, 1999), suggest that colour obsessions and phobias can have a far reaching impact on the behaviour of those with ASD, which in turn could potentially impact on development.

\subsection{Conclusion}

Obsessions and ritualistic behaviours are frequently observed in autism (DSM-IV, 1994) and it is important to understand how extreme reactions to colour relate to obsessions and phobias in general. It is also important to understand the relationship of these atypical reactions to colour to atypical sensory processing in general. It may be the case that those with colour obsessions and phobias, like J.G., also demonstrate atypically severe sensory problems. Further research is needed to investigate these issues, as well as to establish the prevalence of the phenomenon, and the extent to which colour obsessions and phobias ameliorate with development (Dunn, 1999). The current case study provides the first step in understanding colour obsessions and phobias in ASD, and further investigation of this phenomenon could provide insight into the relationship between sensation, perception and behaviour in those with the condition.

\section{Acknowledgements}

We would like to thank all the children for taking part in the study and also their parents and teachers for their cooperation in our research. This work was supported by EU grant (12984) Stages in the Evolution and Development of Sign Use (SEDSU), and a FRSF grant from the University of Surrey to AF.

\section{References}

American Psychiatric Association (1980). Diagnostic and Statistical Manual of Mental Disorders (3 ${ }^{\text {rd }}$. ed.). Washington, DC.

American Psychiatric Association (1994). Diagnostic and Statistical Manual of Mental Disorders (4 ${ }^{\text {th }}$. ed.). Washington, DC.

American Psychiatric Association (2000). Diagnostic and Statistical Manual of Mental Disorders text revision ( $4^{\text {th }}$. ed. $)$. Washington, DC.

Baranek, G. T., Boyd, B. A., Poe, M. D., David, F. J., \& Watson, L. R. (2007). Hyperresponsive sensory patterns in young children with autism, developmental delay, and typical development. American Journal of Mental Retardation, 112, 233-245.

Davies, I., \& Franklin, A. (2002). Categorical similarity may affect colour perception in infants after all. British Journal of Developmental Psychology, 20, 185-203. doi: 10.1348/026151002166406

Dawson, G., \& Watling, R. (2000). Intervention to facilitate auditory, visual and motor integration: A review of the evidence. Journal of Autism and Developmental Disorders, 30, 415-421. doi: 10.1023/A:1005547422749 
Dunn, W. (1999). Sensory Profile. San Antonio, TX: Psychological Corporation.

Fahle, M., \& Poggio, T. (2002). Perceptual Learning. Cambridge, MA: MIT Press.

Filipek, P. A., Accard, O. J., Baranek, G. T., Cook, E.H.,Dawson, G., Gordan, B., Gravel, J. S., Johnson, C. P. L., Kallen, R. J., Levy, S. E., Minshew, N. J., Prizant, B. M., Rapin, I., Rogers, S. J., Stone, W. I., Teplin, S., Tuchman,. R. F., \& Volkmar, F. R. (1999). The screening and diagnosis of Autism Spectrum Disorders. Journal of Autism and Developmental Disorders, 29(6), 439-484. doi:10.1023/A:1021943802493

Fletcher, R. (1998). The City University Colour Vision Test. Third edition. Windsor: Keeler.

Franklin, A., Sowden, P., Burley, R., Notman, L. \& Alder, E. (2008). Colour perception in children wit; h autism. Journal of Autism and Developmental Disorders, 38, 1837-47. doi: 10.1007/s10803-008-0574-6

Franklin, A., Sowden, P., Notman, L., Gonzales-Dixon, M., West, D., Alexander, I., Loveday, S. \& White, A. (2010). Reduced chromatic discrimination in children with Autism Spectrum Disorders. Developmental Science, 13, 188-200. doi: 10.1111/j.1467-7687.2009.00869.x

Franklin, A. \& Sowden, P. (2011). Colour in Autism Spectrum Disorders. In Biggam, Carole P., Hough, Carole, Kay, Christian \& Simmons, David R.C. (Eds.), New Directions in Colour Studies. John Benjamins, Amsterdam, NL, in press.

Fujita, T., Yamasaki, T., Kamio, Y., Hirose, S. \& Tobimatsu, S. (2011). Parvocellular pathway impairments in Autism Spectrum Disorders: Evidence from visual evoked potentials. Research in Autism Spectrum Disorders, 5, 277 285. doi.org/10.1016/j.rasd.2010.04.009

Grandin, T, (1995). Thinking in Pictures: and other reports from life with autism. New York: Doubleday.

Heaton, P., Ludlow, A. K. \& Roberson, D. (2008). When less is more: Poor discrimination but good colour memory in autism. Research in Autism Spectrum Disorders, 2 (1), 147-156. doi.org/10.1016/j.rasd.2007.04.004

Ishihara, S. (1970). Tests for Colour-Blindness. Tokyo: Kanahara Shuppan,38 plates.

Jones, R. S. P., Quigney, C. \& Huws, J. C. (2003). First-hand accounts of sensory perceptual experiences in autism: A qualitative analysis. Journal of Intellectual \& Developmental Disability, 28, 2, 112-121. doi:10.1080/1366825031000147058

Koh, H.C., Milne, E. \& Dobkins, K. (2011). Contrast sensitivity for motion detection and direction discrimination in adolescents with autism spectrum disorders and their siblings. Neuropsychologia, 48, 40464056. doi.org/10.1016/j.neuropsychologia.2010.10.008

Leekam, S. R., Libby, S. J., Wing, L., \& Gould, J. (2007). Describing the sensory abnormalities of children and adults with autism. Journal of Autism and Developmental Disorders,37, 894-910. doi: 10.1007/s10803-006-0218-7

Liss, M., Saulnier, C., Fein, D., \& Kinsbourne, M. (2006). Sensory and attention abnormalities in autistic spectrum disorders. Autism, 10, 155-172. doi: $10.1177 / 1362361306062021$

Ludlow, A. K. \& Wilkins, A. J. (2009). Case report: Colour as a therapeutic intervention. Journal of Autism and Developmental Disorders, 39, 815-818. doi: 10.1007/s10803-008-0672-5

Minshew, N. J., \& Hobson, J.A. (2008). Sensory sensitivities and performance on sensory perceptual tasks in highfunctioning individuals with autism. Journal of Autism and Developmental Disorders, 38(8), 1485-1498. doi: 10.1007/s10803-007-0528-4

Moore, S. (2004). George and Sam. London: Viking.

Munsell, A. H. (1905). A Color Notation. Boston: Ellis.

Ornitz, E. M. (1989). Autism at the interface between sensory and information processing. In G, Dawson, Autism: Nature, Diagnosis, and Treatment, 174-207. New York: The Guildford Press.

Palmer, S. E., \& Schloss, K. B. (2010). Anecological valence theory of human colour preference. Proceedings of the National Academy of Sciences, 107(19), 8877-8882. doi: 10.1073/pnas.0906172107

Raven, J. C. M., Court, J. H., \& Raven, J. (1992). Standard Progressive Matrices. OUP: Oxford Psychologists Press.

Snodgrass J. G., \&Vanderwart M. (1980) A standardized set of 260 pictures: norms for name agreement, image agreement, familiarity, and visual complexity. Journal of Experimental Psychology and Human Learning, 6, 17421. doi: 10.1037/0278-7393.6.2.174

Tomchek, S. D., \& Dunn, W. (2007). Sensory processing in children with and without autism: a comparative study using the short sensory profile. American Journal of Occupational Therapy. 61, 190-200. doi: 10.5014/ajot.61.2.190

Wechsler D., Golombok S., \& Rust J. (1992). WISC-III Wechsler Intelligence Scale for Children.( $3^{\text {rd }}$ ed.). UK Manual. Sidcup, UK: The Psychological Corporation.

White, B. B., \& White, M. S. (1987). Autism from the inside. Medical Hypotheses, 24, 223-229. doi.org/10.1016/03069877(87)90068-5

Williams, D. (1999). Like Colour to the Blind. London: Jessica Kingsley Publishers Ltd. 


\section{Endnotes}

The Munsell system is a standardised color metric which defines color along three dimensions: hue; chroma (roughly equivalent to saturation or intensity); and value (lightness). Hues in the metric are denoted by a letter (e.g., $\mathrm{R}=$ 'red') and a number $(2.5,5 ., 7.5$ or 10$)$. CIE (1931) Y,x,y color space defines color in terms of its luminance $(\mathrm{Y})$ and how red-green (x) and blue-yellow (y) the colors are.

One possibility is that atypical chromatic processing in ASD is restricted to higher spatial frequencies (stimuli in Fujita et al. were of a higher spatial frequency to those in Koh et al.) or to processing of chromatic stimuli that are static.

Inspection of the data confirmed that J.G.'s error in the blue region was not due to him choosing only one of the blues (e.g., his favourite blue) every time.

$$
\text { Appendix }
$$

Colour Preference Questionnaire

1) Does your child have any favourite colours and if so which ones?

2) Why does your child say they like them?

3) How long have they liked these colours?

4) Does your child have any strong dislikes to certain colours and if so which ones?

5) Why does your child say they dislike them?

6) How long have they disliked these colours?

7) Does your child's like of certain colours influence their behaviour and if so how?

8) Does your child's like of certain colours influence their behaviour and if so how? 